\title{
BM]
}

\section{Effectiveness of paramedic practitioners in attending 999 calls from elderly people in the community: cluster randomised controlled trial}

\author{
Suzanne Mason, reader in emergency medicine, ${ }^{1}$ Emma Knowles, research fellow, ${ }^{1}$ Brigitte \\ Colwell, research associate, ${ }^{1}$ Simon Dixon, senior lecturer, ${ }^{3}$ Iim Wardrope, consultant in emergency \\ medicine, ${ }^{2}$ Robert Gorringe, lead emergency care practitioner, ${ }^{4}$ Helen Snooks, professor of health services \\ research, ${ }^{5}$ Julie Perrin, nurse consultant in emergency medicine, ${ }^{2}$ Jon Nicholl, professor ${ }^{1}$
}

'Health Services Research, School of Health and Related Research, University of Sheffield,

Sheffield S1 4DA

${ }^{2}$ Department of Emergency Medicine, Sheffield Teaching Hospitals Trust, Sheffield S5 7AU

${ }^{3}$ Health Economics and Decision Science, School of Health and

Related Research, University of Sheffield, Sheffield

${ }^{4}$ South Yorkshire Ambulance Service, Rotherham S60 2BO

${ }^{5}$ Centre for Health Information Research and Evaluation, School of Medicine, Swansea University, Swansea SA2 8PP

Correspondence to: $\mathrm{S}$ Mason s.mason@sheffield.ac.uk

doi:10.1136/bmj.39343.649097.55

\section{ABSTRACT}

Objective To evaluate the benefits of paramedic practitioners assessing and, when possible, treating older people in the community after minor injury or illness. Paramedic practitioners have been trained with extended skills to assess, treat, and discharge older patients with minor acute conditions in the community. Design Cluster randomised controlled trial involving 56 clusters. Weeks were randomised to the paramedic practitioner service being active (intervention) or inactive (control) when the standard 999 service was available.

Setting A large urban area in England.

Participants 3018 patients aged over 60 who called the emergency services ( $n=1549$ intervention, $n=1469$ control).

Main outcome measures Emergency department attendance or hospital admission between 0 and 28 days; interval from time of call to time of discharge; patients' satisfaction with the service received.

Results Overall, patients in the intervention group were less likely to attend an emergency department (relative risk $0.72,95 \%$ confidence interval 0.68 to 0.75 ) or require hospital admission within 28 days $(0.87,0.81$ to 0.94$)$ and experienced a shorter total episode time ( $235 \mathrm{~V}$ 278 minutes, $95 \%$ confidence interval for difference 60 minutes to -25 minutes). Patients in the intervention group were more likely to report being highly satisfied with their healthcare episode (relative risk $1.16,1.09$ to 1.23). There was no significant difference in 28 day mortality $(0.87,0.63$ to 1.21$)$.

Conclusions Paramedics with extended skills can provide a clinically effective alternative to standard ambulance transfer and treatment in an emergency department for elderly patients with acute minor conditions. Trial registration ISRCTN27796329.

\section{INTRODUCTION}

The UK Department of Health's strategy has been to encourage the increased use of non-medical staff to carry out assessments and treatments traditionally carried out by doctors. ${ }^{1}$ The introduction of new models of care, including further assessment, triage, and treatment skills for paramedics, has been recommended to help manage ever increasing demands for health care. ${ }^{2}$ Current evidence concerning safety, effectiveness, and costs to support these changes in practice, however, is lacking. ${ }^{3}$

Paramedics can be trained to assess and treat or refer patients with a range of conditions such as wounds, ${ }^{4}$ hypoglycaemia, ${ }^{5}$ falls, and epistaxis. ${ }^{6}$ The merits of a pre-hospital practitioner working in certain geographical areas such as rural locations in fulfilling a broader public health and primary care outreach role in the local community have also been discussed. ${ }^{7}$ Other authors, however, have cast doubt on the safety, feasibility, and cost effectiveness of paramedics assessing and treating apparently minor problems in the community. ${ }^{89}$

Elderly people make $12-21 \%$ of visits to emergency departments. Many of them attend after an accident or fall. ${ }^{1011}$ Recently completed studies suggest that an alternative approach to an emergency ambulance response would have the greatest chance of improving patients' experience, as well as potentially helping to reduce demand, if it was targeted at elderly patients with minor complaints. ${ }^{12} 13$

The South Yorkshire Ambulance Service developed the paramedic practitioner in older people's support (PPOPS) scheme to deliver patient centred care to elderly people who call the emergency services with conditions triaged as not immediately life threatening. Practitioners underwent a three week full time theory based course with lectures from specialists in emergency medicine or care of the elderly. They spent a period of 45 days in supervised practice.

Seven experienced paramedics were selected through open competition and completed the training course to enable them to provide community based clinical assessment for patients aged over 60 who contacted the emergency ambulance service with minor acute conditions. Initial assessment and, when appropriate, treatment was delivered within the patient's residence by an individual paramedic practitioner who responded to emergency calls. When the 
paramedic practitioner deemed it necessary, patients were transported to an emergency department for further assessment or treatment such as radiological investigation. ${ }^{14}$ The box outlines the scope of practice.

Operational between the hours of $8 \mathrm{am}$ and $8 \mathrm{pm}$ each day, the service was activated by a 999 call or an urgent call from a general practitioner to the ambulance control room or from an ambulance crew attending an eligible patient.

We conducted a cluster randomised controlled trial to evaluate the effectiveness and safety of this new service.

\section{METHODS}

Patients were recruited from 1 September 2003 to 26 September 2004. Patients aged 60 and above were eligible for inclusion when the call to the ambulance service originated from a Sheffield postcode between $8 \mathrm{am}$ and $8 \mathrm{pm}$, with a presenting complaint that fell within the scope of practice of the paramedic practitioners. We used cluster randomisation to reduce the risk of contamination (practice in the control group being influenced by the presence of the paramedic practitioner in the community) and to allow service level, rather than individual patient level, evaluation of the intervention. Weeks were randomised before the start of the study (to allow for rostering of the paramedic practitioners) to the paramedic practitioner service being active (intervention) or inactive (control), when the standard 999 service was available. The forward roster was concealed from other members of the emergency services. During inactive weeks, the paramedic practitioners were removed from operational duties

\section{Scope of practice of paramedic practitioners}

\section{Presenting complaint}

- Falls

- Lacerations

- Epistaxis

- Minor burns

- Foreign body in ear, nose, or throat

Practical skills

- Local anaesthetic techniques

- Wound care and suturing techniques

- Principles of dressings and splintage

Special skills

- Joint examination

- Examination of neurological, cardiovascular, and respiratory system

- Examination of ear, nose, and throat

- Protocol led dispensing: simple analgesia, antibiotics, tetanus toxoid

- Assessment of mobility and social needs

Additional options for referral and requesting investigations

- Requests for radiography

- Referral processes: emergency department, general practitioner, district nurse, community social services within the ambulance service, and undertook research duties including obtaining patients' consent and follow-up. Randomisation of weeks was undertaken by computer random number generation.

Before the trial we carried out a four week pilot study to establish the number of weeks needed to complete recruitment and to test data collection methods.

Principal outcomes in the study protocol were attendance at emergency department and hospital admission between 0 and 28 days, interval from time of call to time of discharge, and patients' satisfaction with the service received. Secondary outcomes were investigations and treatments prescribed, subsequent use of health services within 28 days, and health status and mortality at 28 days.

\section{Recruitment of patients}

During each week, a paramedic practitioner based in the ambulance control room identified eligible calls by the presenting complaint and notified a paramedic practitioner in the community (during intervention weeks) or in the emergency department (during control weeks). All identified patients were approached face to face either in the community or in the emergency department for written consent to follow-up. To avoid unnecessary burden on participants, patients who had more than one eligible episode were recruited only for their first episode.

If patients were unable to complete questionnairesfor example, because of cognitive impairment or who were unable to read English - we obtained consent for follow-up by review of clinical records only.

The research team independently checked the ambulance service call database at the end of each month for any additional eligible calls not identified by the paramedic practitioners at the time of the incident. We noted patients identified retrospectively to check for selection bias but did not follow them up.

\section{Data collection}

\section{Routine data}

The research team used the emergency department or ambulance service records to collect clinical data, including investigations, treatment, diagnoses, and discharge from the service, relating to the initial patient episode. Total episode time was derived by calculating the interval between the time the initial call was received in the ambulance control room to the time that the patient left the emergency department, was admitted to hospital, or, if the patient was discharged in to the community, the time that the paramedic practitioner or ambulance crew left the scene. These times therefore included any time spent waiting for assessment in the emergency department.

We used hospital records to collect information about unplanned hospital attendances or admissions within Sheffield in the 28 days after the initial episode and mortality at 28 days. Information relating to subsequent ambulance requests was collected from the local ambulance service. Attendance at an emergency department or hospital admission on day 0 was 
combined with any unplanned attendances at an emergency department or admissions in the 28 days that followed to provide information on overall unplanned use of hospital services.

\section{Survey of patients}

Follow-up was by postal questionnaire at three and 28 days after the incident. The three day questionnaire asked patients about examinations, treatments, advice, and satisfaction with the service they had received. One of our primary outcome measures of patients' satisfaction was based on one question asking about overall satisfaction with the care received during the initial episode and was measured on a five point scale.

The 28 day questionnaire contained items on subsequent use of health services relating to the incident and perceived change in physical health and included the general health status measure, the EQ-5D. ${ }^{15}$

\section{Sample size}

We calculated our sample size on the basis of four primary outcomes: satisfaction with care, attendance at emergency department, hospital admission, and total episode time. The number of primary outcomes reflects the importance of considering different aspects of the impact of service delivery on patients and services in a pragmatic multi-dimensional study. If, as we expected, there is no clustering of data in relation to these outcomes within weeks, we needed about 1100 patients in each group to have an $80 \%$ chance of detecting as significant at the $5 \%$ level a $5 \%$ change in the proportion of "very satisfied" patients. If there is clustering, with an intraclass cluster correlation of 0.02 and 40 patients per cluster, this sample size gives $80 \%$ power to detect a difference of $75 \%$ versus $82 \%$. If we ignore the clustering, this sample size also gives $80 \%$ power to detect a change of $4 \%$ in the proportion of patients attending the emergency department, a change of $6 \%$ in the proportion of patients admitted, and a difference of 20 minutes in the mean total episode time (assuming an SD of 180 minutes).

On the basis on the results of the four week pilot study, to recruit two sets of 1100 patients to follow-up we randomly allocated 52 weeks, later extended to

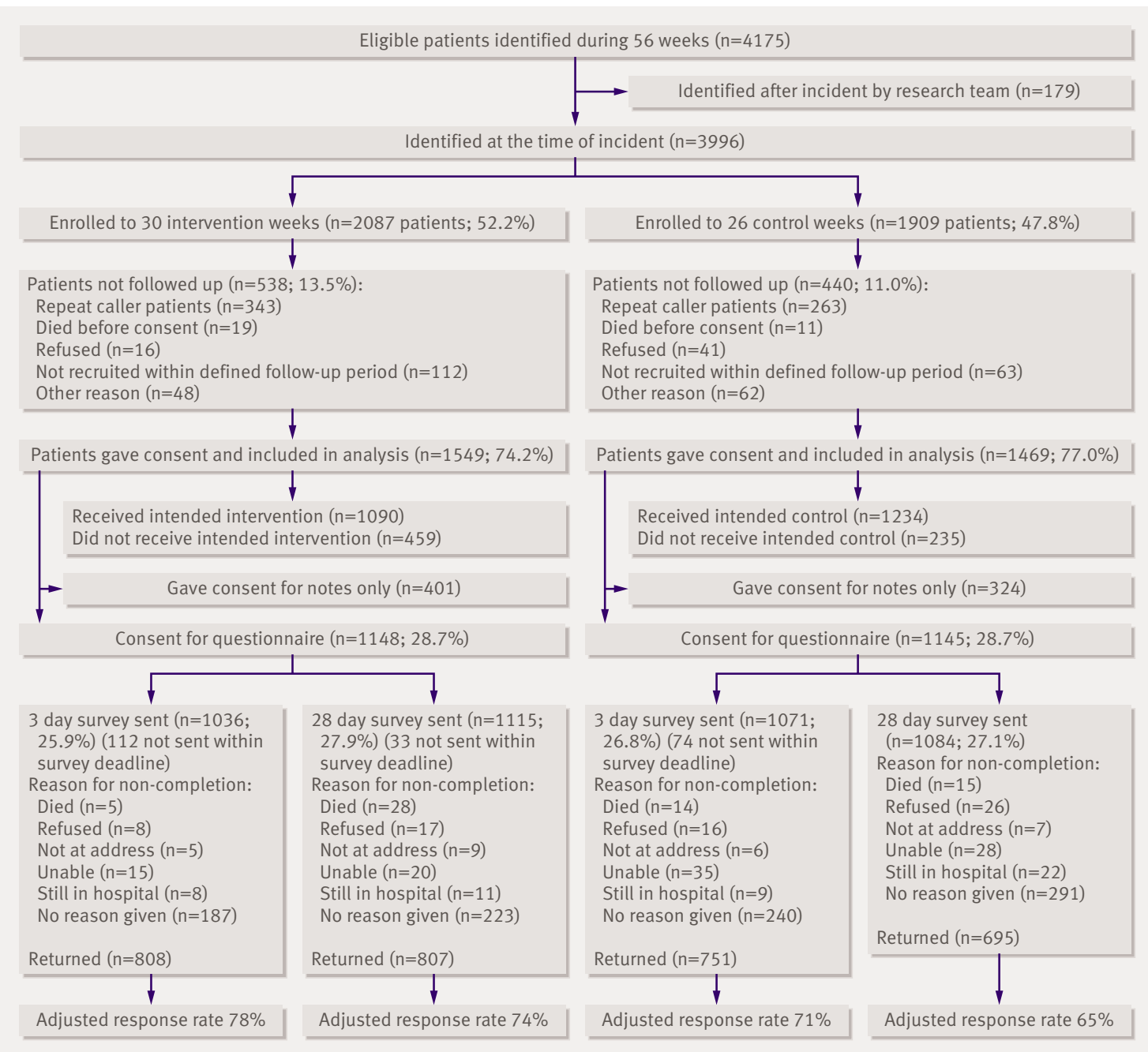


Table 1 | Baseline characteristics of all patients identified

\begin{tabular}{lccc} 
& $\begin{array}{c}\text { Identified by paramedic } \\
\text { practitioner }(\mathrm{n}=3996)\end{array}$ & $\begin{array}{c}\text { Not identified by paramedic } \\
\text { practitioner }(\mathrm{n}=179)\end{array}$ & Total $(\mathrm{n}=\mathbf{4 1 7 5 )}$ \\
& $2877(72.0)$ & $123(68.7)$ & $3000(71.9)$ \\
\hline Women (\%) & $82.52(8.38)$ & $79.17(9.14)$ & $82.37(8.44)$ \\
\hline Mean (SD) age (years) & $3529(88.3)$ & $156(87.2)$ & $3685(88.3)$ \\
\hline Presented after fall (\%) & &
\end{tabular}

${ }^{*} \mathrm{P}=<0.001$.

56 weeks, to achieve the sample size. The 56 weeks were randomly allocated in an unrestricted design into control $(n=26)$ and intervention $(n=30)$ weeks.

\section{Statistical analysis}

Analysis was by randomisation, on an intention to treat basis, irrespective of the actual service received. During the intervention weeks, identified patients should have received an assessment by a paramedic practitioner in the community. This was not always possible if a paramedic practitioner was busy assessing another case when an eligible call was received. Such patients were attended by a standard emergency ambulance response and, according to ambulance service protocols, should have been taken to the emergency department by ambulance unless they refused transport. During the control weeks, identified patients were attended and treated according to standard practice as described above.

SPSSv.12 was used for initial statistical analysis of baseline differences between groups. Data were then exported to STATAv.8.0 to enable analysis at a cluster level. ${ }^{16}$ Generalised estimating equations were used to correct the standard errors of control and intervention comparisons for the effect of any correlation within weeks. To allow for a proper estimation of a relative risk, we used a Poisson error distribution with a robust standard error. ${ }^{1718}$

\section{RESULTS}

\section{Trial numbers}

During the trial, the paramedic practitioners identified $96 \%(3996 / 4175)$ of all eligible calls at the time of the incident (figure). There were no significant differences in terms of sex and presenting complaint between those identified by the paramedic practitioner and those identified retrospectively by the research team. Those identified by the paramedic practitioner, however, were a little older than those who were not identified (table 1).

Of the 2087 patients identified during the intervention weeks and 1909 during the control weeks, 978 patients did not consent to participate, resulting

Table 2 | Baseline characteristics of patients identified by paramedic practitioner at time of incident

\begin{tabular}{lccc} 
& Recruited $(\mathrm{n}=\mathbf{3 0 1 8})$ & Not recruited $(\mathrm{n}=\mathbf{9 7 8})$ & Total $(\mathrm{n}=\mathbf{3 9 9 6 )}$ \\
Women (\%) & $2192(72.6)$ & $685(70.0)$ & $2877(72.0)$ \\
\hline Mean (SD) age (years) & $82.55(8.32)$ & $82.43(8.57)$ & $82.52(8.38)$ \\
\hline Presented after fall (\%) & $2681(88.9)$ & $848(86.7)$ & $3529(88.3)$ \\
\hline
\end{tabular}

in the inclusion of 3018 patients into the trial. The figure shows details of why patients did not take part. There was a small difference in recruitment rates between intervention (74\%) and control (77\%) weeks, but no significant differences between the baseline demographics of those who were recruited and those who were not (table 2).

During intervention weeks most patients $(n=1090)$ received the intended service (assessment by a paramedic practitioner). The other patients received the standard ambulance response and were $(n=390)$ or were not $(n=69)$ transported to the emergency department. During control weeks all patients received a standard ambulance response (1234), although 235 were not transported to the emergency department.

There were no differences between groups in terms of demographics or presenting complaint (table 3). The presenting complaint was identified as the primary complaint allocated by the call taker to the call that initiated the ambulance response.

\section{Primary outcomes}

Patients in the intervention group were less likely to have attended an emergency department either during the initial episode (day 0 ) or in the next 28 days $(62.6 \%$ $v 87.5 \%, \mathrm{P}<0.001$ ). They were also less likely to have required a hospital admission during the same time period $(40.4 \%$ v 46.5\%, $\mathrm{P}<0.001)$ (table 4). Respondents in the intervention group were more likely to report being "very satisfied" than those in the control group $(85.5 \%$ v $73.8 \%, \mathrm{P}<0.001)$. On average, patients in the intervention group experienced a shorter total episode time by around 42 minutes (235 $v$ 278 minutes, $\mathrm{P}<0.001$ ).

\section{Secondary outcomes}

Investigations received by patients during the trial included radiography, blood and urine tests, and electrocardiography. Patients in intervention weeks were less likely to undergo some form of investigation $(49.7 \%$ v $67.9 \%, \mathrm{P}<0.001)$ but were more likely to receive some form of treatment, including advice $(81.3 \%$ v $72.8 \%, \mathrm{P}<0.001)$.

Patients in the intervention group, however, were more likely to have subsequent unplanned contact with secondary care services, such as the ambulance service, emergency department, or hospital admission, in the 28 days after their initial episode (excluding the initial contact on day 0$)(21.3 \% v 17.6 \%, \mathrm{P}<0.01)$. They we also less likely to report that their physical health had worsened compared with those in the control group $(21.7 \%$ v $25.6 \%, \mathrm{P}=0.13)$. The EQ-5D revealed no significant differences in health outcomes between the two groups.

In the 28 days after their initial episode $142(4.7 \%)$ patients died. There were no significant differences between the two groups in terms of mortality.

\section{DISCUSSION}

This randomised controlled trial evaluated the impact on processes and outcomes of paramedics with 


$\begin{aligned} & \text { Table } \mathbf{3} \text { | Baseline characteristics of recruited patients. Figures are numbers (percentages) of } \\
& \text { patients unless stated otherwise }\end{aligned}$
\begin{tabular}{lccc} 
Intervention $(\mathrm{n}=1549)$ & Control $(\mathrm{n}=1469)$ & Total $(\mathrm{n}=\mathbf{3 0 1 8 )}$ \\
Women & $1115(72.0)$ & $1077(73.3)$ & $2192(72.6)$ \\
\hline Mean (SD) age (years) & $82.6(8.3)$ & $82.5(8.3)$ & $82.6(8.3)$ \\
\hline Living in own home & $1209(78.1)$ & $1139(77.5)$ & $2348(77.8)$ \\
\hline Incident occurred at usual residence & $1336(86.2)$ & $1234(84.0)$ & $2570(85.5)$ \\
\hline Presenting complaint: & $1369(88.4)$ & & \\
\hline Fall & $93(6.0)$ & $7313(89.4)$ & $2682(88.9)$ \\
\hline Haemorrhage & $86(5.6)$ & $78(5.3)$ & $171(5.7)$ \\
\hline Acute medical condition & & $164(5.4)$ \\
\hline
\end{tabular}

extended skills managing patients with acute minor conditions. The service conveyed considerable benefits for patients and the NHS in terms of reduced overall attendances at an emergency department and hospital, shorter episode times, and higher levels of satisfaction among patients. The new service also seems to be safe in that we identified no differences in mortality or health outcomes after 28 days.

More than a quarter $(29.6 \%, \mathrm{n}=459)$ of patients in the intervention group did not receive the paramedic practitioner service. These patients therefore received the "normal service" but were still included in the "intervention" group as the results were analysed on a pragmatic intention to treat basis, reflecting the outcomes that could be expected were the intervention to be introduced more widely, and standard for the reporting of the results of health services research. ${ }^{20}$ This had the effect of considerably weakening the impact of the intervention.

The patients in this trial were categorised as having "minor" conditions at their initial contact with the emergency services. The most common presenting complaint was a fall. Within 28 days of the initial call, however, over $40 \%$ had required a hospital admission and $5 \%$ had died. This highlights the high risk nature of this group of patients. None the less, the service seemed to manage the risk appropriately and identify a group of patients who benefited from management at home.

There is increasing strategic pressure within the NHS to extend this type of approach. In 2003, the changing workforce programme, part of the NHS modernisation agency and the Department of Health, set up 17 initial emergency care practitioner pilot sites. These practitioners are mainly paramedics who receive extended skills training, as did the paramedic practitioners in this study (although for a shorter time period). More work is required to enable identification of patients who can benefit from this level of care rather than a full assessment in an emergency department. ${ }^{21} 22$ Some emergency care practitioner schemes are targeted at different populations and operate in different ways and thus the results of this study may not be fully transferable.

\section{Limitations}

This large open pragmatic trial has some limitations because of differences in recruitment of patients and response rates to follow-up questionnaires between the groups. In particular, the measurement of patients' satisfaction depended on receipt of a three day followup questionnaire. Of the 3996 patients randomised to the trial, only 2293 agreed to receive a questionnaire. This was mainly because of the proportion of patients with cognitive impairment, who we excluded from this part of the study. Of the 2293 patients, 1482 (64.6\%) responded, which is less than the number we calculated we needed $(n=2200)$. The effects on the primary outcomes (hospital attendance and admission, episode times, and satisfaction), however, were all significant and sufficiently large for us to be confident that the effects are real.

The study was conducted in one large urban area of the UK. Therefore the generalisability of these results

Table $4 \mid$ Primary and secondary outcomes in patients seen by paramedic practitioners or not. Figures are numbers (percentages) unless stated otherwise

\begin{tabular}{|c|c|c|c|c|c|}
\hline & $\begin{array}{l}\text { Intervention } \\
\text { weeks }\end{array}$ & Control weeks & $\begin{array}{l}\text { Relative risk } \\
(95 \% \mathrm{Cl})\end{array}$ & P value & ICC \\
\hline \multicolumn{6}{|l|}{ Primary outcomes } \\
\hline ED attendance $0-28$ days $(n=3018)$ & $970(62.6)$ & $1286(87.5)$ & $0.72(0.68$ to 0.75$)$ & $<0.001$ & 0.00 \\
\hline Hospital admission $0-28$ days $(n=3018)$ & $626(40.4)$ & $683(46.5)$ & $0.87(0.81$ to 0.94$)$ & $<0.001$ & 0.00 \\
\hline Very satisfied with care $(n=1482)$ & $656(85.5)$ & $528(73.8)$ & $1.16(1.09$ to 1.23$)$ & $<0.001$ & 0.00 \\
\hline Mean (SD) total episode time $(\mathrm{min})(\mathrm{n}=2968)$ & $235.1(183.3)$ & $277.8(182.6)$ & $\begin{array}{c}-42.2(8.8)^{*}(-59.5 \\
\text { to }-25.0)\end{array}$ & $<0.001$ & - \\
\hline \multicolumn{6}{|l|}{ Secondary outcomes } \\
\hline Investigation at initial episode (n=2946) & $754(49.7)$ & $971(67.9)$ & 0.73 (0.69 to 0.78$)$ & $<0.001$ & 0.00 \\
\hline Treatment initial at episode $(n=2946)$ & $1233(81.3)$ & $1040(72.8)$ & $1.11(1.06$ to 1.17$)$ & $<0.001$ & 0.00 \\
\hline $\begin{array}{l}\text { Subsequent unplanned contact with secondary care after initial } \\
\text { episode }(n=3018)\end{array}$ & $330(21.3)$ & $259(17.6)$ & $1.21(1.06$ to 1.38$)$ & $<0.01$ & 0.00 \\
\hline Physical health worse $(n=1430)$ & $166(21.7)$ & $170(25.6)$ & 0.85 (0.69 to 1.05$)$ & 0.13 & 0.00 \\
\hline Mortality at 28 days $(n=3018)$ & $68(4.4)$ & $74(5.0)$ & $0.87(0.63$ to 1.21$)$ & 0.41 & 0.00 \\
\hline
\end{tabular}

ICC=intraclass correlation; $E D=$ emergency department.

${ }^{\star}$ Difference (SE). 


\section{WHAT IS ALREADY KNOWN ON THIS TOPIC}

Paramedics can be trained to manage certain medical conditions outside hospital

They have also been trained to make triage decisions

\section{WHAT THIS STUDY ADDS}

Paramedics can be trained to see and treat elderly people with acute minor conditions and reduce the need for emergency department attendance by almost $25 \%$

Patients find this approach more satisfactory than attending the emergency department should be treated with some caution. We think that there is nothing unique about the patients or presenting complaints. Other health communities could replicate this model, and we are aware of similar services being set up in the UK and abroad. This does require major cooperation between organisations and considerable training and operational costs.

We acknowledge that there may have been some clustering at a practitioner level. Though our study was designed specifically to assess clustering by week, statistical software does not allow for cluster analysis of two variables simultaneously so we could not analyse clustering at a practitioner level.

\section{Summary}

Paramedics with extended skills working in the community can provide a clinically effective alternative to standard ambulance transfer and treatment in an emergency department for elderly patients with acute minor conditions.

Contributors: SM and JW initiated and designed the study. EK oversaw the data collected by BC. JN provided advice on design and statistical advice. SM and EK analysed the data. All authors contributed to the advancement of the project by providing specialist knowledge at study meetings and provided input in re-drafting the manuscript. SM drafted the first manuscript and wrote the final version, which was seen and approved by all of the authors. SM is guarantor. Funding: Health Foundation.

Competing interests: None declared.

Ethical approval: North Sheffield research ethics committee.

Provenance and peer review: Not commissioned; externally peer reviewed.

1 Department of Health. The NHS plan. London: Department of Health, 2001.
2 Department of Health. Taking health care to the patienttransforming NHS ambulance services. London: Department of Health, 2005.

3 Snooks H, Williams S, Crouch R, Foster T, Hartley-Sharpe C, Dale J. NHS emergency response to 999 calls: alternatives for cases that are neither life threatening nor serious. BMJ 2002;325:330-3.

4 Hale D, Sipprell K. Ability of EMT-Bs to determine which wounds can be prepared in the field. Prehosp Emerg Care 2000;4:245-9.

5 Lerner EB, Billittier AJ 4th, Lance DR, Janicke DM, Teuscher JA. Can paramedics safely treat and discharge hypoglycaemic patients in the field? Am J Emerg Med 2003;21:115-20.

6 Snooks H, Kearsley N, Dale J, Halter M, Redhead J, Cheung WY. Towards primary care for non-serious callers to the emergency ambulance service: results of a controlled study of 'Treat and Refer' protocols for ambulance crews. Qual Saf Health Care 2004;13:435-43.

7 O'Meara P. Would a prehospital practitioner model improve patient care in rural Australia? Emerg Med J 2003;20:199-203.

8 Kamper MM, Mahoney BDM, Nelson SR, Peterson J. Feasibility of paramedic treatment and referral of minor illnesses and injuries. Prehosp Emerg Care 2001;5:371-8.

9 Wolford RW, Tisol W, Vasilenko P, Domeier RM, Bignall Owensby S Prospective evaluation of criteria allowing paramedics to treat and release patients presenting with hypoglycaemia. Prehosp Disaster Med 1996;109:S36.

10 Sun BC, Adams JME, Orav JE, Rucker DW, Brennan TA, Burstin H. Determinants of patient satisfaction and willingness to return with patient care. Ann Emerg Med 2000;35:426-34.

11 Castillo PA, Pousada L. Emergency services used by elderly individuals. Clin Geriatr Med 1993;9:491-7.

12 Snooks H, Dale J, Kearsley N, Halter M, Redhead J. Development and impact of emergency ambulance 'Treat and Refer' protocols for nonserious 999 patients. London: London Ambulance Service, 2001.

13 Marks PJ, Daniel TD, Afolabi O, Spiers G, Nguyen-Van Tam JS. Emergency (999) calls to the ambulance service that do not result in the patient being transported to hospital: an epidemiological study. Emerg Med / 2002;19:449-52.

14 Mason S, Wardrope J, Perrin J. Developing a community paramedic practitioner intermediate care support scheme for older people with minor conditions. Emerg Med J 2003;20:196-8.

15 Dolan P. Modeling valuations for EuroQol health states. Med Care 1997;35:1095-108.

16 StataCorp. Statistical software: release 8.0. College Station, TX: Stata Corporation, 2003.

17 Campbell MJ. Statistics at square two. 2nd ed. London: BMJ Books, 2006.

18 Zou G. A modified Poisson regression approach to prospective studies with binary data. Am J Epidemiol 2004;159:702-6.

19 Campbell MK, Elbourne DR, Altman DG. CONSORT statement: extension to cluster randomised trials. BM/ 2004;328:702-8.

20 Schwartz D, Lellouch J. Explanatory and pragmatic attitudes in therapeutic trials. J Chronic Dis 1967;20:637-48.

21 Mason S, Coleman P, O'Keeffe C, Ratcliffe J, Nicholl J. The evolution of the emergency care practitioner role in England: experiences and impact. Emerg Med J 2006;23:435-9.

22 Squires JP, Mason S. Developing alternative ambulance response schemes: analysis of attitudes, barriers, and change. Emerg Med / 2004;21:724-7.

Accepted: 8 August 2007 